\title{
Research on Compartment Positioning Algorithm for the Intelligent Sampling Control System
}

\author{
Ping Xie \\ North China Electric Power University, Beijing, China \\ xieping@ncepu.edu.cn
}

\begin{abstract}
This paper proposes a new algorithm to position the area of a truck's compartment in images for the intelligent sampling control system. The goal is to identify a truck's compartment and assure that some works are done inside it. For example, white a mechanical arm automatically snatches goods carried by a truck, we must ensure that the golods must be obtained inside the truck's compartment. Firstly, the compartment image is acquired by the camera overhead. Secondly, the Canny operator is used to extract the edge of the image, because it is sensitive to the image texture features. Thirdly, the Canny edge image is transformed to the Hough transform space. The peak points in the Hough parameter space indicate the straight lines which may be the elements of the compartment. The straight lines which meet the conditions of the actual sizecof the compartment make up the area of the compartment. Finally, converting the image coordinate to the physical compartment coordinate. The experiment result shows that the algorithm is effective and the coordinate conversion error analysis indicates that the conyerting algorithm is workable.
\end{abstract}

Keywords: edge detecting, Holngh transform, compartment positioning, image processing, intelligent sampling

\section{Introduction}

The mechanical sampling devices have been increasingly applied in various fields to take out samplers for further evaluating or testing. In coal-fired power plants or steel mills, coal is the important raw material and plays a decisive role to the products' quality. For small or medium-sized enterprises, coal is carried into the factory mainly by truck. While picking up the coal samples for further testing to confirm the grade of coal, the mechanical sampling devices are unable to avoid the interference of man-made factors, because the sampling locations are decided by the operation personnel [1]. In order to eliminate the influence of the human faetor and improve the reliability of sampling system, an intelligent sampling control system is necessary. On this situation, the automatic sampling system should be designed. The most important thing in automatic sampling system is identify the area of a truck's compartment. Only the compartment has been identified, the mechanical sampling devices can complete the work correctly.

For the moment, there are mainly two kinds of technologies suitable for compartment positioning. One is the infrared signals localization and ultrasonic positioning that based on sensor; the other is the digital image processing technology which extracts image edge information. The positioning technology based on the sensor is vulnerable to environmental factors, but the positioning technology based on digital image processing can overcome these problems. 
Owing to the compartment is rectangle, many rectangle image identification methods are involved. References [2-6] had discussed image identification issues of straight line and rectangle. For the truck's compartment positioning, reference [7] proposed a locating method based on background-subtraction and image projection method; reference [8] presented an edge detection algorithm on fuzzy sets according to the requests of determining orientation of coal compartment. It is applicable to the edge detection of bigger objective area (compartment) of multi-levels image.

Although the truck's compartment is rectangle, but the location of the truck (in the detection area of the driveway) is not fixed while waiting for sampling, and the size of the compartments vary from a truck to another. Consequently, the key problem for intelligent automatic sampling is how to obtain the locations of the compartments. Hence, those methods described in references must be improved and combined to apply to the truck's compartment ${ }^{\circ}$ positioning.

\section{Sampling Workflows Analysis}

As shown in Figure 1, the truck carries the coal into the factory The RFID (Radio Frequency IDentification) tags system identifies it firstly. Every truck which wants to enter the factory must apply for registration and own a RFID tag. Only those trulcks that have valid RFID tags can enter the sampling area. Secondly, the RFID system checks out the truck's information from its database, such as its number, weight, length, width, height, and so on. After that, the truck enters the sampling area to be sampled. The mechanical sampling device must pick up the samplers correctly inside the compartment. In order to obtain the fair sampler data, the sampling location should be as random as possible, and the samplers should come from multiple locations.

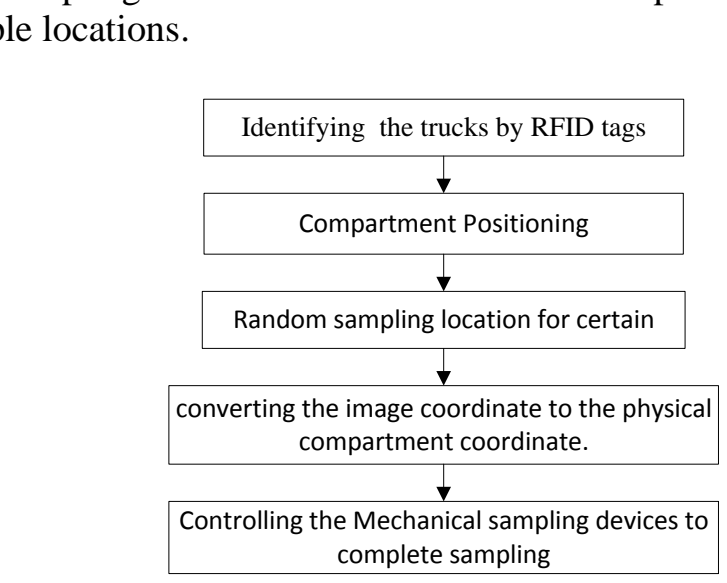

\section{Figure 1. The workflows for intelligent sampling control system}

In order to get the location of the truck in real time, a camera is set up in the top middle of the sampling area as shown in Figure 2. The truck image information is acquired from the dynamic video after the truck has stopped in the sampling area. According to the image characteristics that the color for the truck's compartment filled with coal is black, the image processing technique is used for the image edge detection to calculate the edge of the truck. But the image edge contains the front part of the truck. In order to assure that the sampling locations are inside the compartment, the rectangle compartment area that indicates the truck's compartment should be identified. 


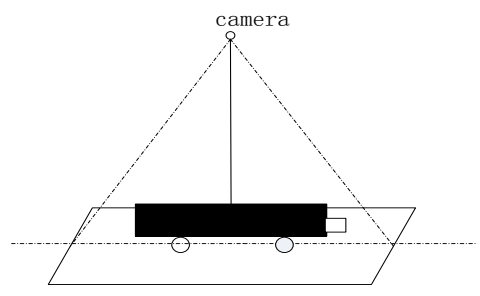

Figure 2. The diagram for sampling area

In image processing, the edge of an image is the boundary among different regions. Roberts operator, Sobel operator, Grad operator, Prewitt operator, Log operator, Laplacian operator and Canny operator are familiar edge detection operators. These operator can ${ }^{\circ}$ identify the boundary in pixel level. For an original image that based on pixel level, the image edge can be detected by the variation on the direction of first-order or second-order defivative between the neighboring regions. Many scholars have made the analysis and comparison among their respective advantages and disadvantages [10]. The edge of the conpartment has obvious characteristic that the coal is black, so the outline of the truck can be asily got with an edge detection algorithm. The Canny operator is used in this paper because it is sensitive to the image texture features.

Due to the imperfections in either the image data or the edge detector, some points or pixels in the desired lines may be lost and the nojsy edge points may appear while obtaining the edge from the Canny operator. However, the Hough transform can address this problem by making it possible to perform groupings ofedge points into line candidates by performing an explicit voting procedure over a set of parameterized image objects. The Hough transform is a feature extraction technique used in image analysis. Computer vision, and digital image processing. It has higher reliability, and dan outputthe ideal result even under the condition of noise, deformation or some loss of image.

The length, width and height data of the truck's compartment is known. The data can be retrieved directly from the truck's RFID tags system, because only those trucks that have valid RFID tags can enterthe sampling area. The sampling area is a one-way road. The trucks must comply with the regulations that they can only move from one side to another. Consequently, the direction of the truck is fixed. We just need to identify the tail location of the truck, and then the whole position of the truck compartment can be labeled with the rectangle geometry constraints

\section{Related Algorithms}

\subsection{Canny edge detection algorithm}

Canny edge detection operator was put forward by John F. Canny in 1986. It is based on the optimization algorithm. The edge of an image is the areas where brightness changes significantly. The gray level in these areas is generally regarded as a step. That is, the gray scale in a small area shows dramatic changes. Canny operator has a higher fidelity on the edge of original image, and also has better edge location accuracy. Hence the Canny operator is adopted.

The Canny edge detection operator has three criteria [9].

(1) SNR (Signal Noise Ratio) criterion. The goal for SNR is to find out all pixels that are part of the edge and exclude the pixels that do not belong to the edge. If expressed in a formula, SNR can be written as the following formula. 


$$
S N R=\frac{\left|\int_{-\infty}^{\infty} G(-x) f(x) d x\right|}{\delta \sqrt{\int_{-\infty}^{\infty} f^{2}(x) d x}}
$$

In formula (1), $f(x)$ is the filter impulse response in the boundary of $[-\infty, \infty], G(x)$ is the edge, $\delta$ is the root mean square for Gauss noise.

(2) L (Localization accuracy) criterion. L criterion shows that the edge pixels found out must coincide with the actual edge as closely as possible. If expressed in a formula, $\mathrm{L}$ can be written as the following formula.

$$
L=\frac{\left|\int_{-\infty}^{\infty} G^{\prime}(x) f^{\prime}(x) d x\right|}{\delta \sqrt{\int_{-\infty}^{\infty} f^{\prime 2}(x) d x}}
$$

In formula (2), $G^{\prime}(x)$ is the first-order derivative of $G(x)$, and $f^{\prime}(x)$ is the firstorder derivative of $f(x)$.

(3) Single edge response criteria. A single edge, has onlyone response, and the false edge response should be restrained as much as possible. It can be expressed with D (the average Distance of the false edge) as follws.

$$
D=\pi \sqrt{\int_{-\infty}^{\infty} f^{\prime 2}(x) d x}
$$

For the three criteria, SNR ariterion reflects the detection rate of an edge detection operator upon the real edge. The higher the SNR, the more likely the detection result contains the real edges and exclude the false edges. L criterion is on behalf of the mathematical expectation between the detection result and the inverse of the actual edge. The Lower the L, the higher the localization accuracy. $D$ shows the average distance of the false edges by the convolution between the random noise and the detection function. The higher the $\mathrm{D}$, the lower possibility the false edges are.

In this paper, the average distance D is considered as a constant, and the optimal product of SNR and $\mathrm{L}$ wich calculated in limited times are considered as Canny detection function, and then it is expressed as the first-order derivative of Gauss function. If expressed in a formula, the Gauss function is $G(x)=e^{-\alpha^{2} x^{2} / 2}$, then the Canny operator can be written as:

$$
C a(x)=x e^{-\alpha^{2} x^{2} / 2}
$$

\subsection{Hough transform algorithm}

The Hough transform used universally today was invented by Richard Duda and Peter Hart in 1972, and they called it a "generalized Hough transform". ${ }^{[1]}$ The classical Hough 
transform was concerned with the identification of lines in the image, but later the Hough transform has been extended to identifying positions of arbitrary shapes. The Hough transform has higher reliability and can bring out the ideal lines even under the condition of noise or some loss of image. The purpose of the Hough transform is to find imperfect instances of lines by a voting procedure. This voting procedure is carried out in a parameter space, from which the line candidates are obtained in a so-called accumulator space that is explicitly constructed by the algorithm for computing the Hough transform.

The basic idea to detect straight lines by Hough transform is the duality principle of points and lines. In other words, the Hough transform changes the problems of detecting straight lines in the image space into detecting points in the Hough transform space. In the image space, a straight line usually can be expressed as $y=k x+b$ that has constant slope $\mathrm{k}$ and intercept $b$. This is called the slope-intercept model of a straight line. In the Hough transform. ${ }^{\bullet}$ Duda and Hart proposed a pair of parameters denoted as $(r, \theta)$ to describe the lines for computational reasons. The pairs $(r, \theta)$ define a polar coordinate. The parameter $r$ repsesents the distance between the line and the coordinate origin, while $\theta$ is the angle of the vector from the coordinate origin to the closest point of the line. Using this parameterization, the equation of the line can be written as the following equation.

$$
r=x \cos \theta+y \sin \theta
$$

(5)

The Hough transform algorithm uses a two-dimensionat array $C(r, \theta)$, called an accumulator, to detect the existence of a line. For each edge pixel $(x, y)$, the Hough transform algorithm determines if there is a straighte line at that pixel. If so, it will calculate the parameters $(r, \theta)$ of that line, and then acumulate it to the corresponding element in the array $C(r, \theta)$. Figure 3 demonstrates hou a line in the image space is mapped to a point in the Hough transform space. In the Hough transform space, there are lots of lines across a point because the parameters $(r, \theta)$ can take various value. But each points of the same line must have a same pair of parameters $(r, \theta)$, and accumulate it into the array $C(r, \theta)$. The final result of the Hough transform is the array $C(f, \theta)$. Each element in the array has a value equal to the number of pixels that are positioned on the line.

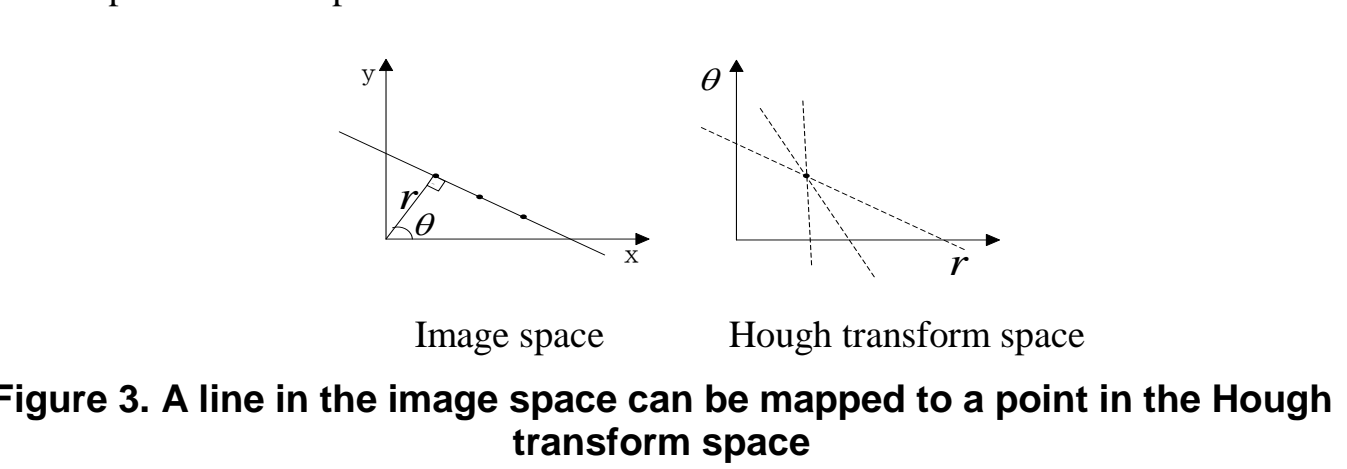

This example illustrates a thing that every point inside the straight line in the image space can be mapped to the same point in the Hough transform space because they have the same slope and intercept. According to the idea of Duda and Hart, any straight line in the original image space can be mapped to a point in the Hough transform space. So the element of array $C(r, \theta)$ with the higher value indicates the straight lines that are most represented in the input image. The length of the straight line segment determines the total value of the 
element of array $C(r, \theta)$. In this way, the problem of detecting straight lines can be replaced with finding peak points in the Hough transform space.

Consider the rectangle shown in Figure 4. $P_{1}\left(x_{1}, y_{1}\right), P_{2}\left(x_{2}, y_{2}\right), P_{3}\left(x_{3}, y_{3}\right)$ and $P_{4}\left(x_{4}, y_{4}\right)$ are the four vertices of the rectangle. The length of sides are $a$ and $b$.

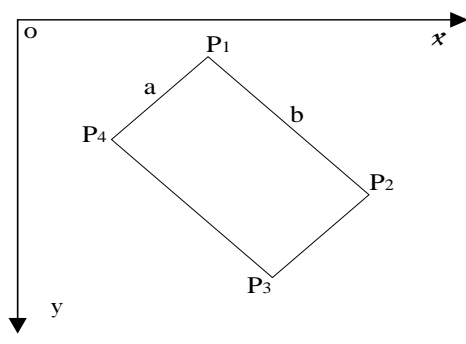

Figure 4. A rectangle in the image

After accumulating the parameters $(r, \theta)$ of the image shown in figure 4 into the corresponding element of array $C(r, \theta)$, the final result of array $C(r, 0)$ is shown in figure 5 , there are 4 peak points after the Hough transform. They are respectively, corresponding to the four sides of the rectangle: $P_{2} P_{3}, P_{4} P_{1}, P_{3} P_{4}$ and $P_{1} P_{2}$.

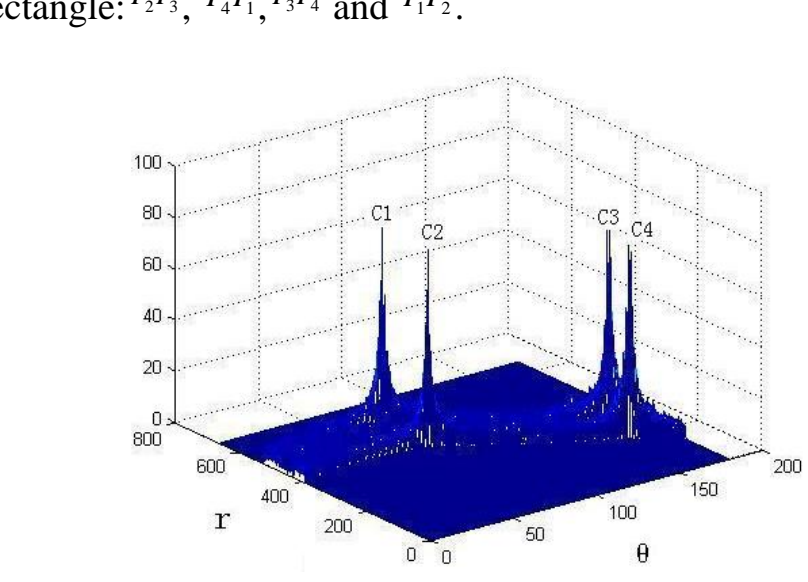

Figure 5. The rectangle in the Hough transform space

The four peak points $C_{1}\left(r_{1}, \theta_{1}\right), C_{2}\left(r_{2}, \theta_{2}\right), C_{3}\left(r_{3}, \theta_{3}\right)$ and $C_{4}\left(r_{4}, \theta_{4}\right)$ ) meet the following conditions:

- The are paired. $C_{1}$ and $C_{2}$ are a pair which meet the condition of $\theta_{1}=\theta_{2}, C_{3}$ and $C_{4}$ are another pair which meet the condition of $\theta_{3}=\theta_{4}$.

- The gap of the parameter $\theta$ between the two pairs of peak points is $90^{\circ}$, in other words, it means $\left|\theta_{1}-\theta_{3}\right|=90^{\circ}$ and $\left|\theta_{2}-\theta_{4}\right|=90^{\circ}$.

- The length of opposite sides is equal, so that the same pair of peak points' accumulating value is equivalent. In other words, it means $C_{1}=C_{2}$ and $C_{3}=C_{4}$.

- The parameter $r$ between the same pair of peak points is related to the length of the rectangle's sides, in other words, it means $\left|\rho_{1}-\rho_{2}\right|=b$ and $\left|\rho_{3}-\rho_{4}\right|=a$. 


\section{Compartment Positioning Algorithm}

\subsection{Algorithm idea}

The length, width and height data of the truck's compartment is known from the truck's RFID tags system, and the direction of the truck is fixed. We just need to identify the tail location of the truck, and then the whole poison of the truck compartment can be calculated with the rectangle geometry constraints.

As long as the tail part of the compartment is identified (In other words, a pair of vertices and three sides of the rectangle are found out), the rectangle compartment area excluding the front part of the truck can be identified according to the length and width data of the compartment come from the RFID tags system. Hence, we just need to cope with a half of the truck image. The compartment positioning algorithm idea is listed as follows.

Step1: Acquire the truck image by the camera set up in the middle of the sampling area.

Step2: Extract the edge of the truck image by Canny edge detectípn operatør.

Step 3: Cope with half of the Canny edge image to perform Hough transform echnique.

Step 4: Extract all existent straight lines in Hough transform space which would belong to the tail edge of the truck.

Step 5: Delete those straight lines which do not satisfy the Sspecific conditions. So the straight lines which may not be part of the tuck's compartment would be removed. Theoretically, there are three straight lines lefte

Step 6: Find out the pair of vertiees which indicates the tail of the truck's compartment region. The vertices are just two intersection points of the three straight lines.

Step 7: Use the rectangle geometric constraints to calculate the other two vertices. Since the four vertices of the rectangle compartinent have been identified, the compartment area also can be confirmed.

Step 8: Convert the inage coordinate to the physical compartment coordinate.

Step 9: Send the coordinate varue to the mechanical sampling devices.

In the following subsections, some of these steps will be described in detail.

\subsection{Straight line extraction algorithm}

In order to find of the straight lines, the Canny edge image should be mapped into the Hough transform space according to the idea of Duda and Hart. The Hough transform is used for the whole image edge, so the range of $r$ is related to the diagonal length of the image, the range of $\theta$ should be $0 \sim 180^{\circ}$. The two-dimensional array $C(r, \theta)$ is used to cumulate the number of those pixels in the Canny edge that meet the straight line equation (5). The elements of $C(r, \theta)$ with the higher value indicate the straight lines that are most represented in the input image. The specific process for straight lines extraction is as follows.

(1) For each edge pixels $(x, y)$ in Canny edge, each $\theta$ can calculate a corresponding value $r$. The $\theta$ may take over any possible value in the range of $0 \sim 180^{\circ}$, each $r$ and $\theta$ constitute the only straight line in the Canny image. The values of array $C(r, \theta)$ are initialized as 0 and accumulated according to the value of $r$ and $\theta$, so we can get the numbers of pixels meet the 
same parameters $r$ and $\theta$, and these elements of $C(r, \theta)$ with the higher value indicates the straight lines in the input image.

(2) Finding out the peak points in the array $C(r, \theta)$ because each peak point is corresponding to a straight line. Method for finding the peak point is to extract all points which meet the threshold condition $C(r, \theta) \geq T_{c}$, and the threshold $T_{c}$ is associated with the actual compartment size. The actual compartment size was known, so the amount of lines extracted can be limited in a certain range.

\subsection{Vertex extraction algorithm}

We have known that $C_{1}\left(r_{1}, \theta_{1}\right), C_{2}\left(r_{2}, \theta_{2}\right), \cdots, C_{n}\left(r_{n}, \theta_{n}\right)$ are $\mathrm{n}$ peak points extracted from ${ }^{\circ}$ array $C(r, \theta)$ by previous straight line extraction algorithm, the goal for this step is to find ont a pair of vertices (that is, two intersection points of three straight lines) which ind cates the tail of the truck's rectangle compartment region.

Firstly, we must find out a pair of parallel sides belong to the compartment by scanning each peak point. If a pair of peak points which meet the following condition then they would be made a pair.

$$
\left|\theta_{i}-\theta_{j}\right|<T_{\theta} \text { and }|| \rho_{i}-\rho_{j}|-W|<T_{w}
$$

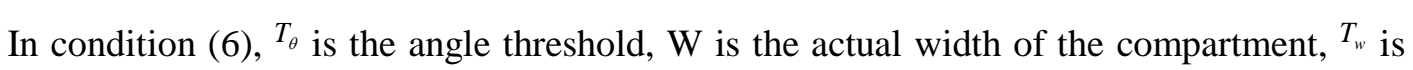
the width deviation threshold.

Among those peak points which dre not paired successfully, the third side of the compartment $C_{k}\left(\rho_{k}, \theta_{k}\right)$ is just the gne which satisfres the following condition.

$$
|| \theta_{k}-\frac{\theta_{i}+\theta_{j}}{2}\left|-90^{2}\right|<\hat{T}_{\alpha}
$$

In condition (7), is the angle threshold.

Now the three peak points we found out just represent the three sides of the tail compartment. The rest work is to extract the two vertices of the tail compartment.

Assuming the three peak points we find out are $C_{1}\left(r_{1}, \theta_{1}\right), C_{2}\left(r_{2}, \theta_{2}\right)$ and $C_{4}\left(r_{4}, \theta_{4}\right)$ as shown in Figure 5. As ancexample, $C_{1}$ and $C_{4}$ are involved to demonstrate how to get a vertex. The intersection point of the two straight lines is exactly the vertex wanted.

We can get equation (8) by feeding the parameters $C_{1}\left(\rho_{1}, \theta_{1}\right)$ into equation (5) and get equation (9) by feeding the parameters $C_{4}\left(\rho_{4}, \theta_{4}\right)$ into equation (5).

$$
\begin{aligned}
& \rho_{1}=x \cos \theta_{1}+y \sin \theta_{1} \\
& \rho_{4}=x \cos \theta_{4}+y \sin \theta_{4}
\end{aligned}
$$

The intersection point of the two straight lines can be calculated by equation (8) and (9) and the intersection point is corresponding to the vertex $P_{2}\left(x_{2}, y_{2}\right)$. 
Similarly we can calculate the intersection point of $C_{2}$ and $C_{4}$ which is corresponding to the vertex $P_{1}\left(x_{1}, y_{1}\right)$. So we have known a pair of vertices and three sides of the compartment. It means a pair of vertices and three sides of the rectangle compartment have been found out.

\subsection{Solving rectangle compartment vertices}

The problem of solving rectangle compartment vertices can be changed into solving the location of the vertices ${ }^{P_{3}}$ and ${ }^{P_{4}}$ shown in Figure 4, because we have known the location of vertices $P_{1}, P_{2}$, three edges, and the length of $\mathrm{a}$ and $\mathrm{b}$ in Figure 4.

The three peak points of the rectangle have been known as $C_{1}\left(\rho_{1}, \theta_{1}\right), C_{2}\left(\rho_{2}, \theta_{2}\right)$ and $C_{4}\left(\rho_{4}, \theta_{4}\right)$. According to the relationship among the four Hough peak points described in Section 3 , the ${ }^{\circ}$ last peak point $C_{3}\left(\rho_{3}, \theta_{3}\right)$ should meet the condition of $\left|\rho_{3}-\rho_{4}\right|=a$ and $\theta_{3}=\theta_{4}$. The verter $P_{3}$ is exactly the intersection point of $C_{1}$ and $C_{3}$, and the vertex $P_{4}$ is exactly the intersection point of $C_{2}$ and ${ }^{C_{3}}$. Hence, refer to the equation (5) we can work out thelm. Now, the four vertices have been identified, the location of the compartment is just the area by connecting the for vertices.

\section{Experiment Result}

Firstly, the Canny operator is used to detect the edge of the Original image. The original image acquired by the camera overhead is shown in Figure 6, the Canny edge image is shown in Figure 7. The front part of the truck is included in the Canny) edge.

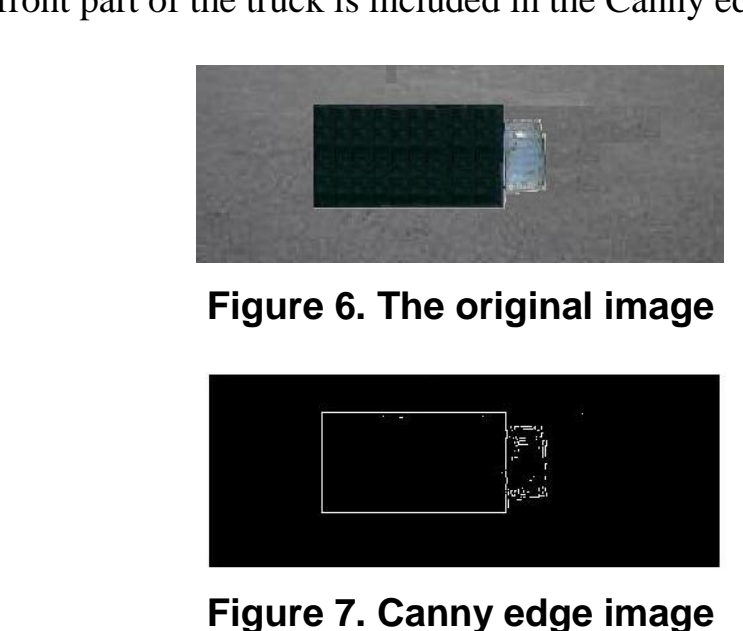

Secondiy, Cutting out half of the Canny edge image to perform rectangle feature extraction with the xindowed Hough transform algorithm. Each peak point in the Hough transform space is corresponding to a straight line. The tail part of the compartment includes 3 lines, and the 3 straight lines extracted are shown in Figure 8.

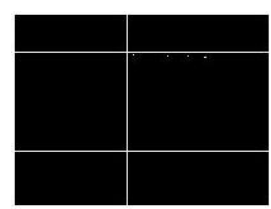

Figure 8. The straight lines extracted in the tail part 
Thirdly, scanning each peak point to find out the pair of parallel edges which satisfy condition (6) and the third edge of the compartment which satisfies condition (7). According to equation (8) and (9), we can calculate two intersection points related with the 3 straight lines shown in Figure 8.

Then, according to the relationship among the four Hough peak points described in section 3 , the fourth characteristic straight line of the compartment can be obtained. So we can get the other two vertices by finding out the intersection points among the fourth straight line and the two parallel lines. As shown in Figure 9, the rectangle region of the compartment is the area marked by connecting the four vertices.

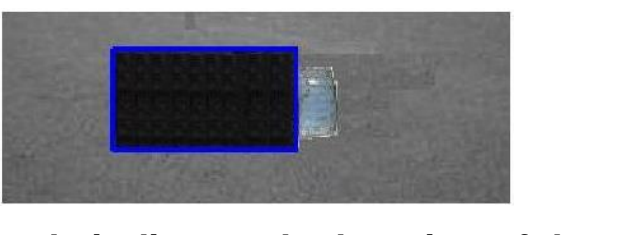

Figure 9. The rectangle indicates the location of the compartment

Finally, converting the image coordinate to the physical compartment coordinate.

\section{Conversion Error Analysis}

The image coordinate is in pixels, converting the inage coordinate to the physical compartment coordinate is the premise condition to ensure that the coal samples must be obtained inside the truck's compartment.

\subsection{Converting algorithm}

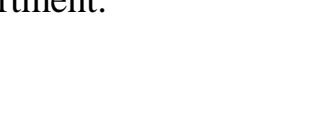

As shown in Figure 10, supposing the top view angle of the camera is $\varphi$, the distance from the ground to the camera is $H$, and the sizo of the image is $640 \times 480$.

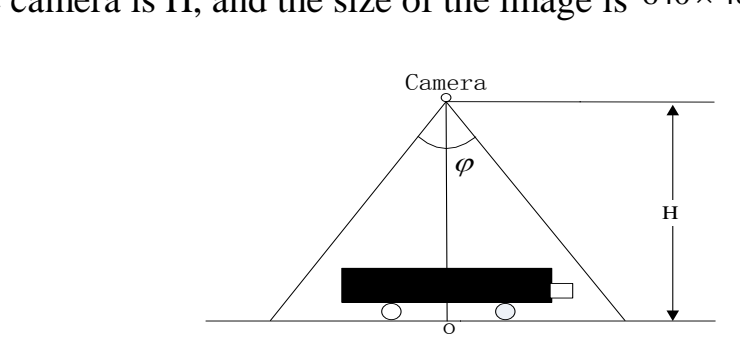

\section{Figure 10. Converting the coordinate}

(1) Scaling. Considering each degree of $\varphi$ includes the same number of pixels, so a pixel can be converted to $\frac{\varphi}{640}$ degree in the horizontal orientation, and the length for a pixel is $L_{h 0}=\operatorname{tg}\left(\frac{\varphi}{640}\right) \times H$. Similarly, a pixel can be converted to $\frac{\varphi}{480}$ degree in the vertical orientation, and the length for a pixel is $L_{v 0}=\operatorname{tg}\left(\frac{\varphi}{480}\right) \times H$.

(2) Conversion. Considering a random pixel ${ }^{(x, y)}$, firstly, converting to $\left(x_{0}, y_{0}\right)$ that relative to the image center point; and then converting to the physical compartment coordinate $\left(P X_{0}, P Y_{0}\right)$ that relative to the physical center point $\mathrm{o}$ according to the ${ }^{L_{h 0}}$ and ${ }^{L 0}$. 


\subsection{Conversion error analysis}

This converting algorithm may introduce mainly two kinds of errors as follows.

(1) The top view angle $\varphi$ of camera and the distance from the ground to the camera $\mathrm{H}$ may introduce error.

As shown in Figure 11, supposing the distance from the ground to the camera is $\mathrm{H}$, the distance from the ground to the truck's roof is $\mathrm{h}$, the physical center point is o. Now, considering the point $P_{1}$. The horizontal coordinate (physical coordinate) of $P_{1}$ relative to the center point $\mathrm{o}$ is $X_{1}$. Due to the fixed angle of camera view, $P_{1}$ is moved to the projection point $P_{2}$ with the horizontal coordinate $X_{2}$ from the camera. $X_{1}$ and $X_{2}$ are related because $X_{2}=X_{1}+h \times \operatorname{tg} \alpha$ and $\operatorname{tg} \alpha=\frac{X_{1}}{H-h}$. Hence, the top view angle $\varphi$ of camera and the distance from the ground to the camera $\mathrm{H}$ introduce the error $h \times \operatorname{tg} \alpha$ in the horizontal coordinate That means $X_{1}<X_{2}$. Similarly, we can derive $Y_{Y_{1}<Y_{2}}$ and so forth.

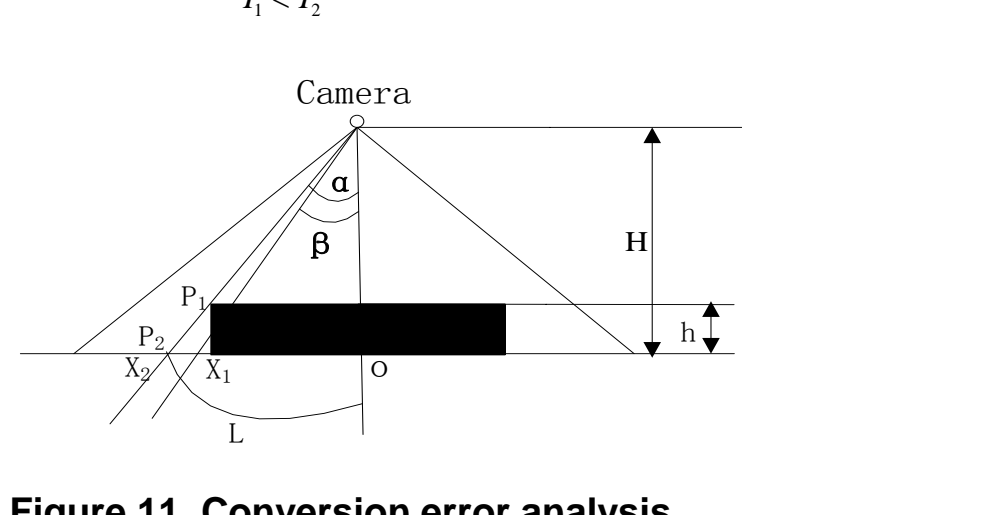

Figure 11. Conversionerror analysis

(2) Scaling formula may introduce error

In Scaling, the number of pixels matched to each degree of $\varphi$ is considered to be equal. However, it is relative to the arc length $\mathrm{L}$ in fact. Hence, for point $P_{1}$, the real horizontal coordinate of the projection poin (4s) $X_{2}=H \times \operatorname{tg} \beta$ and $\beta=\frac{X_{2}}{\text { the radius of circle }}$ after scaling calculation. However, the calculation formula should be $\alpha=\frac{L}{\text { the radius of circle }}$ in fact. It means $X_{2}{ }^{\prime}<X_{2}$. Similarly, we can also derive, $Y_{2}^{\prime}<Y_{2}$.

In conclusion, the two kinds of errors are opposite. They can cancel each other out. The top view angle $\varphi$ of camera and the distance from the ground to the camera $\mathrm{H}$ may expand the error, but the scaling calculation can reduce the error.

\section{Conchusion}

In this paper, a new algorithm is proposed to position the truck's compartment full of coal. In the power plants and steel mills whose main material is coal, the sampling control device must know the sampling locations which are inside the compartment. This algorithm is achieved by Canny edge and Hough transform. Firstly, the truck image is acquired by the camera overhead. Secondly, the Canny operator is used to extract the edge of the image, because it is sensitive to the image texture features. Thirdly, the Canny edge image is transformed to the Hough transform space. The peak points in the Hough parameter space indicate the straight lines which may be the elements of the compartment. The actual size of 
the compartment is introduced into the calculating, hence, the positioning accuracy of the truck can be increased and it is important for the coal sampling automation. Finally, converting the image coordinate to the physical compartment coordinate, and the coordinate conversion error analysis indicates that the converting method is workable.

\section{References}

[1] S. Y. Xie, Z. Y. Zhao, Z. Q. Shang and Z. W. Zhao, "Study on belt samplers for truck-coal in a coal-fired power plant", J. Electric Power, vol. 45, (2012).

[2] S. S. Bao and L. Yang, "Algorithm for detecting vertical and horizontal lines in real-time image processing", J. Journal of Hefei University of Technology, vol. 26, (2003).

[3] Q. Q. Wang, M. Y. Cao, N. L. Sun and D. Fan, “Accurate Localization Algorithm for Rectangle Mark Based on Spatial Interpolation Technology and Combined Detection Method", Proceedings of the 2008 International ${ }^{\bullet}$ Congress on Image and Signal Processing, (2008) May 27-30; Sanya, China.

[4] Z. Yuan and Q. Z. Zhu, "Rectangle detection by the chain-code tracing", Proceedings of the 2nd International Conference on Electrical and Control Engineering, (2011) September 14-18; Yichang, China.

[5] T. T. Nguyen, X. D. Pham and J. W. Jeon, "Rectangular object tracking based on standardhough tyansform", Proceedings of the 2008 IEEE International Conference on Robotics and Biomimetics, (2008) February 2225; Guilin, China.

[6] C. R. Jung and R. Schramm, "Rectangle Detection based on a Windowed Hough Transform", Proceedings of the 2004 IEEE XVII Brazilian Symposium on Computer Graphics and Image Processing, (2004) October 1720; Curitiba, Brazil.

[7] C. Zhou, "Car Position Locating Method Based on Image Processing", J. Compyter systems \& applications, vol. 20, (2011).

[8] Q. X. Yang and H. Y. Wu, "New fuzzy EDA and its application in determining orientation of coal compartment", J. Computer Engineering and Design, vol. 28, (2007).

[9] J. Canny, "A computational approach to edge deteetion", J. IEEE Transactions on Pattern Analysis and Machine Intelligence, vol. 8, (1986).

[10] R. C. Gonzalez, "Digital Image Processing" J. Publishing House of Electronics Industry in press, (2006).

[11] R. O. Duda and P. E. Hart, "Use of the Hough Transformation to Detect Lines and Curves in Pictures", J. Comm. ACM, vol. 15, (1972).

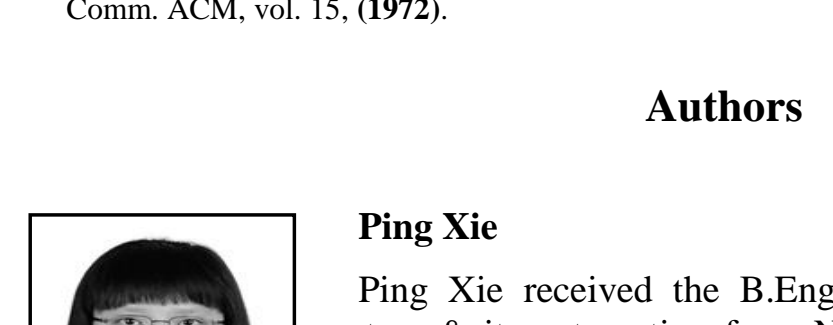

Ping Xie received the B.Eng degree and M.Eng degree in power system \& its automation from North China Electric Power University, China in 1995 and 1998 respectively. She is currently researching on jmage processing and intelligent sampling. 